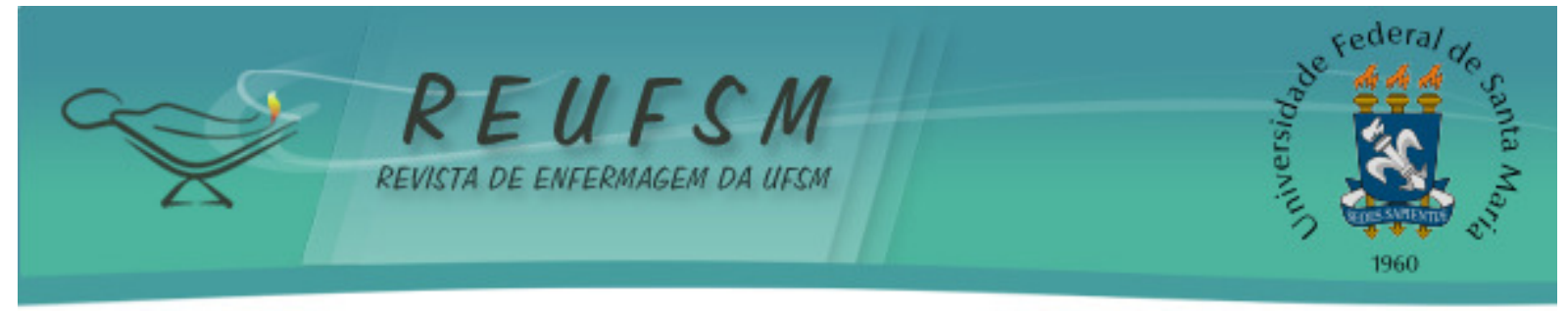

ARTIGO ORIGINAL

\title{
A FORMAÇÃO PROFISSIONAL NA ORIENTAÇÃO DA ASSISTÊNCIA AOS GRUPOS VULNERÁVEIS NA ATENÇÃO BÁSICA
}

\author{
VOCATIONAL TRAINING IN DIRECTING ASSISTANCE TO VULNERABLE GROUPS IN PRIMARY \\ CARE
}

\section{LA FORMACIÓN PROFESIONAL EN LA DIRECCIÓN DE LA ASISTENCIA A LOS GRUPOS VULNERABLES EN LA ATENCIÓN PRIMARIA}

Doi: $10.5902 / 2179769213738$

\author{
Letícia de Lima Trindade ${ }^{1}$ \\ Lucimare Ferraz ${ }^{2}$ \\ Sílvia Fátima Ferraboli ${ }^{3}$ \\ Bruna Rubini ${ }^{4}$ \\ Camila Trevisan Saldanha ${ }^{5}$ \\ Maiara Bordignon ${ }^{6}$ \\ Carine Vendruscolo ${ }^{7}$
}

RESUMO: Objetivo: identificar como os processos de formação profissional e de Educação Permanente em Saúde têm orientado a assistência aos grupos populacionais vulneráveis. Método: pesquisa descritiva e qualitativa, realizada com 15 profissionais de saúde, entre médicos, enfermeiros e técnicos de enfermagem. Utilizou-se da entrevista para coleta de dados e estas foram analisadas na proposta de Bardin. Resultados: houve a contextualização da vulnerabilidade na formação dos profissionais, contudo, majoritariamente de forma teórica e desarticulada da prática. Destacaram a Educação Continuada e Permanente em Saúde como importantes estratégias para qualificação da assistência. Considerações finais: o reconhecimento dos aspectos da formação dos sujeitos que atuam no Sistema Único de Saúde emerge como primordial para uma melhor compreensão das vulnerabilidades no contexto da Atenção Básica em Saúde.

Descritores: Vulnerabilidade em saúde; Atenção primária à saúde; Ensino superior; Educação continuada.

ABSTRACT: Aim: to identify how the processes of training and Continuing Education in Health have guided assistance to vulnerable population groups. Method: descriptive qualitative research, which was attended by 15 health professionals, including physicians, nurses and nursing technicians. The interview was used for data collection and these were analyzed in the proposed Bardin. Results: the vulnerability has been contextualized in the training, however, mostly theoretical and the practical disjointed. Continuing Education and Permanent Health were highlighted as important strategies for upgrading care. Final

\footnotetext{
${ }^{1}$ Enfermeira. Doutora em Enfermagem. Docente na Universidade do Estado de Santa Catarina e Universidade Comunitária Regional de Chapecó. Chapecó, SC, Brasil. E-mail: letrindade@hotmail.com

2 Enfermeira. Doutora em Ciências da Saúde. Docente na Universidade do Estado de Santa Catarina e Universidade Comunitária Regional de Chapecó. Chapecó, SC, Brasil. E-mail: ferraz.lucimare@gmail.com

${ }^{3}$ Enfermeira. Residente na Ênfase de Atenção ao Paciente Crítico na Residência Integrada em Saúde no Grupo Hospitalar Conceição. Porto Alegre,RS, Brasil. E-mail: sil.ferraboli@gmail.com

${ }^{4}$ Graduanda em Enfermagem pela Universidade do Estado de Santa Catarina. Chapecó, SC, Brasil. E-mail: brunexx2@hotmail.com

${ }^{5}$ Enfermeira. Especialista em Gestão em Saúde. Palmitos, SC, Brasil. E-mail: camila_tgremio@hotmail.com

${ }^{6}$ Enfermeira. Mestranda em Ciências da Saúde pelo Programa de Pós-Graduação da Faculdade de Enfermagem da Universidade Estadual de Campinas. Campinas, SP, Brasil. E-mail: bordignonmaiara@gmail.com

${ }^{7}$ Enfermeira. Doutora em Enfermagem. Docente na Universidade do Estado de Santa Catarina. Chapecó, SC, Brasil. E-mail: carineven@yahoo.com.br
} 


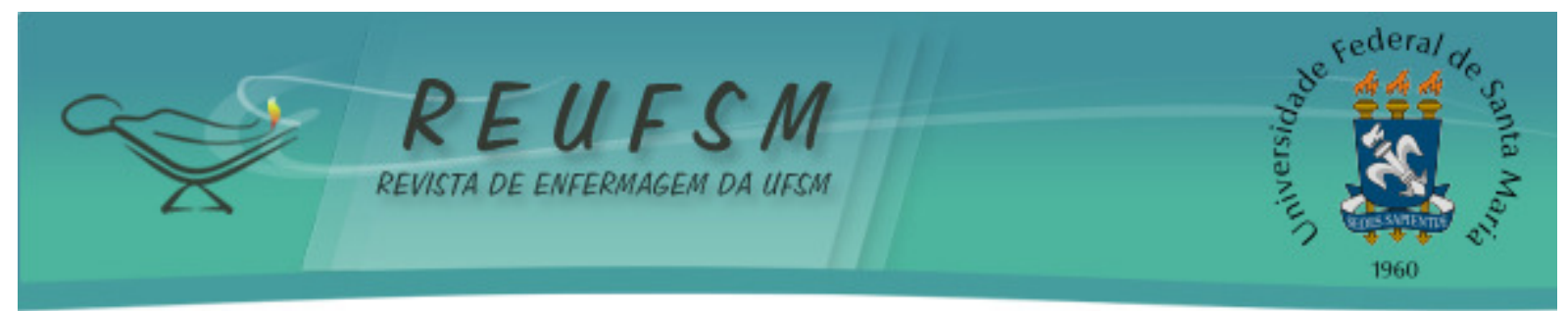

Remarks: the recognition of the aspects of the training of individuals who work in Health System emerged as essential for a better understanding of vulnerabilities in the context of Primary Care in Health.

Descriptors: Health vulnerability; Primary health care; Teaching; Education, higher; Education, continuing.

RESUMEN: Objetivo: identificar como los procesos de capacitación y educación continua en salud han guiado a la asistencia los grupos vulnerables de la población. Método: investigación cualitativa descriptiva, realizada con 15 profesionales de la salud, incluyendo médicos, enfermeras y técnicos de enfermería. Se utilizó la entrevista para recolección de datos, los cuales fueron analizados de acuerdo con Bardin. Resultados: la vulnerabilidad ha sido contextualizado en la formación profesional, sin embargo, en su mayoría de forma teórica, desarticulada de la práctica. Se destacó la Educación Continua y Permanente en Salud como estrategia importante para la mejora de la atención. Consideraciones finales: el reconocimiento de los aspectos de la formación de las personas que trabajan en el Sistema de Salud es esencial para una mejor comprensión de las vulnerabilidades en el contexto de la Atención Primaria en Salud.

Descriptores: Vulnerabilidad en salud; Atención primaria de salud; Enseñanza; Educación superior; Educación continua.

\section{INTRODUÇÃO}

O sistema universitário brasileiro possui 2.252 instituições de ensino superior conforme mostram os dados do Instituto Nacional de Estudos e Pesquisas (INEP), sendo 2.016 instituições privadas e 236 públicas, contabilizando 5.080 .056 alunos matriculados. ${ }^{1}$ Diante disso, é importante refletir como tem ocorrido este processo de formação na área da saúde, uma vez que, o foco da atuação destes profissionais é o ser humano, e para o qual as ações se destinam nos distintos contextos de cuidado.

$\mathrm{Na}$ Atenção Básica $(A B)$ em Saúde, por exemplo, os profissionais desempenham papel crucial no atendimento à população e nas práticas de promoção da saúde, porém a demanda populacional é diversificada, o que exige destes, conhecimentos e métodos diferenciados para prestar assistência adequada.

Ao se trabalhar com grupos populacionais vulneráveis, além de identificar os indivíduos expostos a determinados agravos, observa-se a necessidade de uma assistência singular. Cumpre destacar, que é papel da equipe de saúde identificar esses grupos e os fatores que determinam sua condição de vulnerabilidade, para vinculá-los ao serviço de saúde. $^{2}$

A vulnerabilidade está associada a percepção de que a chance de exposição das pessoas às doenças ou agravos e ao adoecimento, não resulta exclusivamente de um conjunto de aspectos individuais, mas também coletivos e contextuais, além da disponibilidade de recursos para se proteger, caracterizando assim, os indivíduos/grupos em menor ou maior situação de vulnerabilidade. ${ }^{3}$

As constantes alterações econômicas, culturais, políticas e sociais que ocorrem desde o século XIX, causam significativas mudanças na vida da população. Dessa forma, o Brasil vem investindo na elaboração e consolidação de políticas públicas que priorizam a promoção, proteção e recuperação da saúde, visando à melhoria da qualidade de vida e à redução da vulnerabilidade relacionada aos determinantes socioeconômicos, políticos, culturais e ambientais. ${ }^{4}$

Com base no exposto, salienta-se a importância do processo de formação em contemplar as especificidades dos grupos populacionais vulneráveis, como forma de 


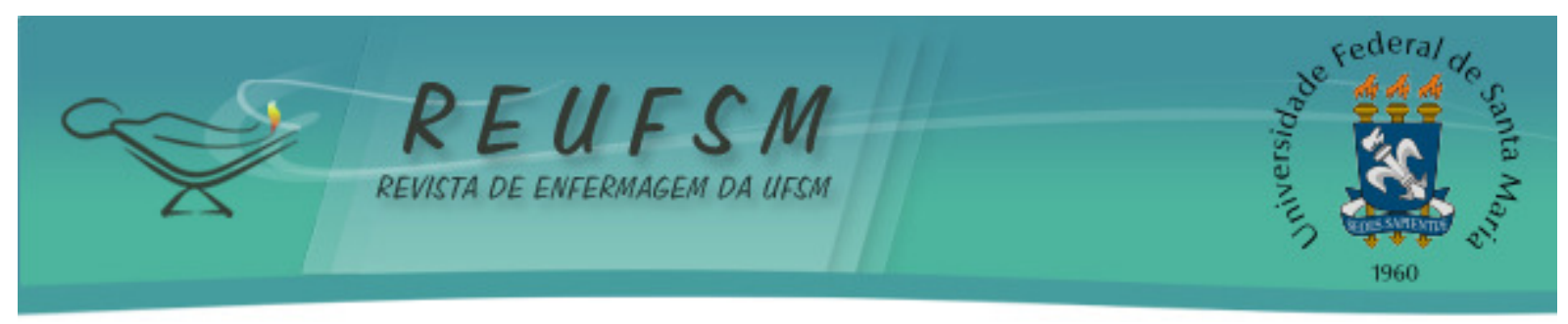

orientar a prática profissional no cuidado a estes indivíduos. Um estudo ao investigar a vulnerabilidade na adolescência na ótica dos enfermeiros da Estratégia de Saúde da Família, evidenciou o despreparo relatado por alguns enfermeiros no que se refere ao assistir integralmente os adolescentes no âmbito da $A B$, que dada as especificidades desta fase da vida, e outros fatores, podem ser considerados sujeitos vulneráveis ao adoecimento e à morte. Esta dificuldade relatada pelos enfermeiros advém, sobretudo, do desconhecimento do modo como interagir com o adolescente e a didática a ser utilizada nas atividades de educação em saúde direcionadas a este grupo. ${ }^{5}$

Assim, mesmo com os progressos na formação dos profissionais de saúde, a necessidade de avançar na direção de um cuidado integral ainda é um desafio, evidenciando a importância de se desenvolver ações de Educação Permanente em Saúde (EPS) ${ }^{6}$ definida como a aprendizagem no trabalho, no qual o aprender e o ensinar são incorporados ao cotidiano laboral e das organizações. Ou seja, propõe que o processo educativo aconteça no local de convivência dos profissionais, com base nos problemas e nos conhecimentos prévios de cada um, tendo como objetivo a transformação de práticas e da dinâmica organizacional, mediante a proposta de problematização do processo de trabalho. ${ }^{7}$ Há a necessidade de atualização constante e espaços para o diálogo, em prol do desenvolvimento de competências profissionais para atuação no Sistema Único de Saúde. ${ }^{8}$ Neste contexto, a EPS busca auxiliar a compreensão dos processos sociais, do cotidiano no qual o indivíduo está inserido ${ }^{9}$, podendo contribuir para a qualificação acerca do cuidado a grupos populacionais específicos, incluindo as populações vulneráveis.

0 presente estudo se propôs a identificar como os processos de formação profissional e de EPS têm orientado a assistência aos grupos populacionais vulneráveis. Compreende-se que investigações sobre esse objeto contribuem para melhor identificar potencialidades e fragilidades inerentes ao processo de formação dos profissionais de saúde, com vistas a promover uma assistência qualificada, dentre outros, aos grupos populacionais mais vulneráveis (idosos, crianças, moradores de rua, índios, etc), no contexto do Sistema Único de Saúde.

\section{MÉTODO}

Trata-se de uma pesquisa descritiva e qualitativa, da qual participaram profissionais de saúde que assistiam populações consideradas vulneráveis em cinco cenários da $A B$ de um município localizado no Oeste do Estado de Santa Catarina, Região Sul do Brasil.

Foram critérios para a inclusão dos profissionais no estudo: 1) ser profissional de saúde de uma Unidade Básica Tradicional (UBT), unidades de Estratégia de Saúde da Família (ESF), sendo uma urbana e uma rural, uma Unidade de Saúde (UBS) em terra indígena, e um Centro de Apoio Psicossocial (CAPS); 2) ser enfermeiro, médico ou técnico de enfermagem, sendo no mínimo um de cada categoria por serviço; 3) fazer parte do quadro de profissionais do serviço, com vínculo empregatício; e 4) atuar no serviço há pelo menos seis meses, acreditando-se que a partir deste tempo, o profissional tende a melhor conhecer o processo de trabalho na unidade em que atua.

0 grupo de participantes compôs-se de 15 profissionais de saúde que prestam assistência às populações vulneráveis no âmbito da $A B$, sendo cinco enfermeiros, cinco médicos e cinco técnicos de enfermagem, distribuídos proporcionalmente entre a Unidade Básica Tradicional (UBT), unidades de Estratégia de Saúde da Família (ESF) rural, urbana e em terra indígena, e Centro de Apoio Psicossocial (CAPS), ou seja, um profissional de cada categoria por cenário de estudo.

As informações foram coletadas por meio de entrevistas semiestruturadas individuais, realizadas no local de trabalho dos profissionais após agendamento prévio, 


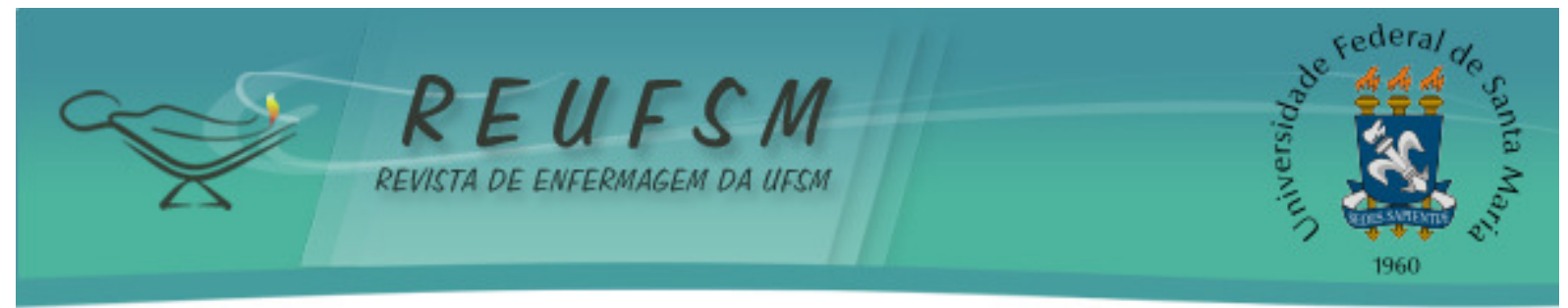

sendo estas registradas em gravador digital e posteriormente transcritas na íntegra. A coleta de dados deu-se no segundo semestre de 2011 e primeiro semestre de 2012.

A análise dos dados ocorreu à luz da Análise Temática de Bardin ${ }^{10}$, compreendendo as suas três etapas: pré-análise, exploração do material, tratamento dos resultados, inferência e interpretação. Inicialmente foi feita leitura flutuante das transcrições, obtendo impressões iniciais acerca do material. Posteriormente, houve a exploração do material, destacando nos relatos dos profissionais os trechos relevantes para o estudo, e por fim, ocorreu a interpretação dos dados a propósito do objetivo e interesses de discussão, com auxílio da literatura científica.

O projeto foi submetido e aprovado pelo ao Comitê de Ética da Universidade do Estado de Santa Catarina (UDESC), sob o número 149.244/2012, e atendeu aos requisitos da Resolução 466/12 do Conselho Nacional de Saúde.

Para preservar o anonimato, os sujeitos foram identificados nesse trabalho pela sigla da categoria profissional (Enfermeiro-E; Médico-M; TE-Técnico de Enfermagem), seguido do número de ordem da transcrição das entrevistas (exemplo: E1, E2, E3...).

\section{RESULTADOS E DISCUSSÃO}

No conjunto dos relatos dos profissionais entrevistados emergiram as seguintes categorias temáticas: a vulnerabilidade no contexto da formação acadêmica; e Educação Permanente em Saúde: fortalecendo a abordagem aos vulneráveis.

\section{A vulnerabilidade no contexto da formação acadêmica}

Identificou-se que, dentre os profissionais de saúde entrevistados, a maioria afirma ter sido abordado em sua formação, algum referencial que trata da vulnerabilidade, sendo discutido tanto de forma teórica quanto prática.

Sim, desde quando nós iniciamos o período da faculdade, depois a gente teve o trabalho em campo [...]. (E1)

Na minha faculdade, a minha foi a segunda turma [...] e a gente começou dentro da comunidade, era um dia, nós já estávamos na comunidade fazendo o cadastramento das pessoas, tipo um agente de saúde faz, conhecendo a realidade de perto, tínhamos reuniões e lá a gente discutia as questões dos adolescentes, formamos grupos, para tratar os adolescentes com relação as drogas, domésticas, idosos [...]. (M3)

Assim, tem se observado a preocupação com a formação de pessoal na área da saúde, em vista à necessidade de formar profissionais para atuar em conformidade com o modelo assistencial proposto pelo SUS e suas demandas, o que vem inclusive, impulsionando a formulação e implementação de políticas públicas. ${ }^{11}$

Em contrapartida, destes profissionais que tiveram a oportunidade de trabalhar a vulnerabilidade em sua formação, alguns contataram o tema majoritariamente de forma teórica.

Se foi discutido foi nos primeiros semestres, teoricamente, e depois na prática isso se perdeu. (M2) 


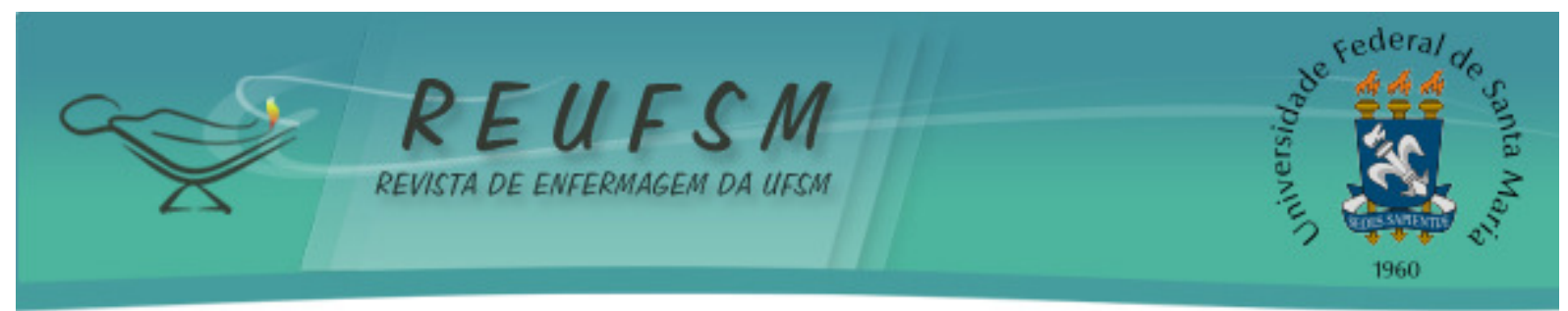

Foram [discutidas as temáticas] através das teorias, assim só. (TE3)

Entende-se que o aspecto teórico ganha significado quando associado à experiência prática, e pode ser concretizado mediante o planejamento e execução de ações. Para os autores, a educação deve garantir a reflexão sobre o que acontece nos serviços, e a aprendizagem precisa ser significativa, promovendo e produzindo sentidos, para que possa assim, efetivamente, promover qualidade na atenção à saúde da população. ${ }^{12}$

Ainda, alguns dos entrevistados afirmaram não ter sido abordada a temática da vulnerabilidade ao longo de seu processo formativo.

Na formação não, só depois de trabalhar. (M5)

Há dezessete anos atrás não, que foi logo que surgiu o SUS então todas estas coisas, era tudo muito novo, não tinha. (E2)

Mais em relação com a população de baixa renda, de criança de alto risco para mortalidade infantil, então muito mais focado na questão da renda, do que na característica, na especificidade de ser indígena [que hoje atuo], ou de ser quilombola, ou de ser negro, esta especificidade não era trabalhada. (M4)

Neste relato, pode-se perceber que algumas especificidades correspondentes a cada grupo populacional, não foram abordadas durante a sua formação. Acredita-se que o enfoque sob o qual a vulnerabilidade deve ser abordada na formação dos profissionais deve envolver a discussão sobre prevenção de doenças e promoção da saúde, com base na realidade dos sujeitos e a partir da aproximação dos profissionais com esses indivíduos, em seu contexto socioeconômico e cultural.

Complementa-se que, no Brasil, é crescente a preocupação de formar profissionais capazes de atuar diante de diversas tecnologias, mas em sua maioria incapazes de lidar com a subjetividade e a diversidade das pessoas que acessam os serviços de saúde. ${ }^{13}$

Quanto ao foco a qual se direcionou a discussão das vulnerabilidades durante a formação profissional, alguns entrevistados referiram que o tema foi pouco abordado por ter sua formação voltada, predominantemente, para a área hospitalar, demonstrando a associação da temática preponderantemente com a AB.

Com certeza não [foi discutida a temática], nessa minha época as escolas de enfermagem tinham a sua formação voltada ao trabalho hospitalar. Não se falava sem saúde pública porque foi bem antes da municipalização da atenção básica. Eu trabalhei vários anos onde não se focava a saúde pública. (TE5)

Infelizmente, eu não tive a oportunidade no curso de visualizar isso, nós fizemos estágio na Saúde Pública, mas um estágio de atendimento e tal [...] daí no hospital, o estágio no hospital não tem né, é mais assistencial mesmo. (TE3)

A gente tinha, na minha época da faculdade, o foco foi muito mais hospitalar, voltado para o cuidado do doente, do que para promoção e prevenção da saúde [...]. (E3) 


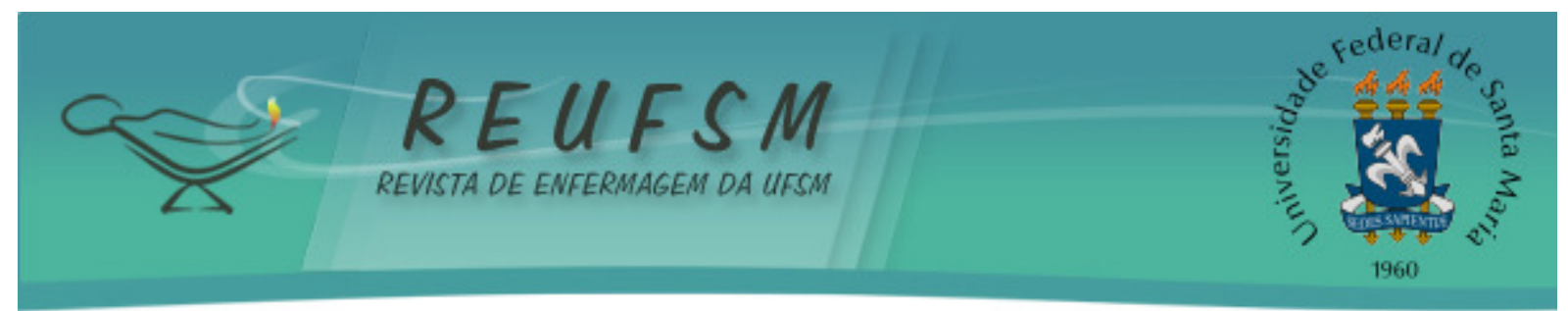

Marcadamente, observa-se a associação da vulnerabilidade à $A B$, revelando a fragilidade da compreensão da temática. Ressalta-se assim, a importância de incluir ou fortalecer este tema, relevante e atual, na formação dos profissionais de saúde. Logo, o conceito de vulnerabilidade pode ser utilizado como parâmetro para formulação de políticas públicas, ${ }^{14}$ assim como na orientação de estratégias que visem a qualidade do serviço prestado.

\section{Educação Permanente em Saúde: fortalecendo a abordagem aos grupos mais} vulneráveis

Entre as estratégias que qualificariam a assistência dos profissionais, os sujeitos mencionaram a Educação Permanente e Continuada em saúde.

Eu acredito muito na questão de educação continuada e não simplesmente chamar alguém lá e se reunir, deveria ter na própria unidade reuniões com a enfermeira, reuniões de equipe para planejar e discutir os casos, quais são os problemas. (TE5)

Penso que basicamente um trabalho assim permanente dos profissionais que trabalham na área de saúde para que eles tenham um aporte assim, um espaço de reflexão, um espaço de discussão, para que outras cabeças pensem juntos no sentido de poder criar realmente força, juntar força de forma complementar para trabalhar com essas populações de risco, penso que para isso seria fundamental a educação permanente. (M1)

Essas ações se fazem relevantes a medida que estes trabalhadores devem desenvolver aptidões para atuar de modo crítico e criativo, buscando ações humanizadas e que visem solucionar os problemas, mediante atividades de prevenção de doenças e agravos, promoção da saúde, recuperação e reabilitação. Logo, o conceito de vulnerabilidade no campo da saúde é recente, e relaciona-se às ações preventivas e às possibilidades de avançar para implementação de práticas que superem o modelo biomédico e a utilização corriqueira de estudos epidemiológicos apoiados, unicamente, no risco. ${ }^{15}$ Além disso, cabe aos profissionais de saúde realizar ações de planejamento, organização, operacionalização e avaliação da efetividade das atividades desenvolvidas à população. ${ }^{16}$

Nessa direção, cumpre ainda refletir sobre as divergências conceituais quando se trata da abordagem de processos de educação permanente e/ou educação continuada. A proposta de EPS do Ministério da Saúde contrapõe-se ao conceito de educação continuada, que se relaciona às capacitações pós formação inicial, com o objetivo de estruturação dos serviços de saúde. Por outro lado, com a EPS, procura-se manter o vínculo entre formação e exercício da profissão, por meio de novas metodologias de ensino e de aprendizagem, em especial, a problematização e a aprendizagem significativa. ${ }^{17}$

Neste estudo, identificou-se na maioria dos relatos dos participantes a existência de processos de qualificação/capacitação a fim de assistir populações vulneráveis. Entretanto, encontram dificuldades em participar de processos educativos, que ocorrem além do âmbito do local de trabalho, pelo fato de não haver profissionais que supervisionem e assumam as atividades da unidade durante sua ausência.

Os profissionais destacaram ainda, participar continuadamente por meio de reuniões de equipe e capacitação externa ou ao longo do ano, de qualificações e 


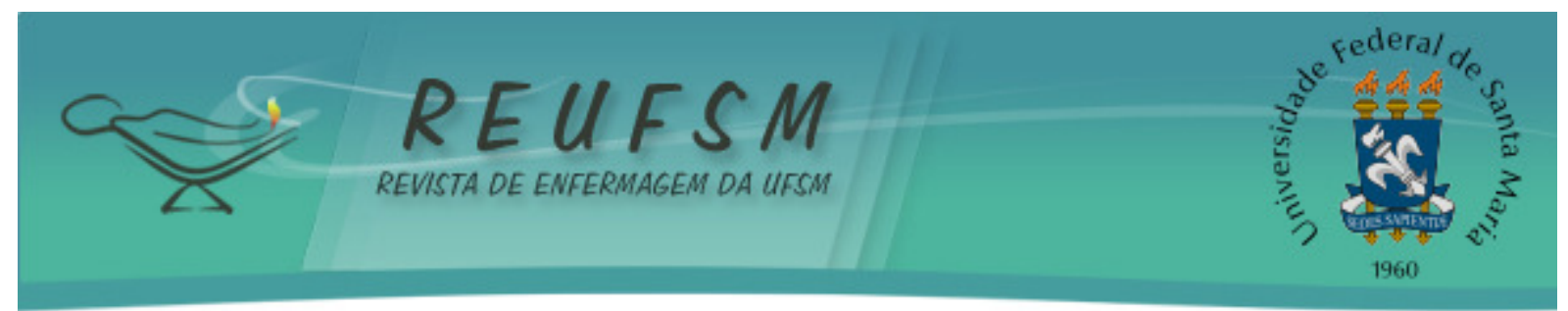

atualizações. Contudo, também mencionaram a falta de periodicidade destas atividades para as equipes. Deste modo, a falta de pessoal em número adequado e a descontinuidade das atividades, constituem-se em empecilhos para o trabalhador.

[...] eu que fico sozinha à tarde, eu costumo faltar bastante no Posto à tarde, porque a gente sempre tem reuniões só a tarde, as capacitações são geralmente a tarde, então o Posto fica descoberto nesta hora. (E4)

Então você tem isso, o Ministério da Saúde preconiza, mas às vezes o próprio Ministério da Saúde não te dá as condições. (E1)

Acredita-se que as atividades de qualificação dos profissionais devem ocorrer periodicamente, mediante métodos de reflexão e participação ativa, gerando maior qualificação e motivação, bem como, expectativa para contribuir com a tomada de decisões e nos resultados da unidade, e deste modo, fazer o uso de todas as habilidades e potencial dos profissionais da equipe. ${ }^{18}$ Para que isso ocorra, é imprescindível realizar a escolha adequada do tema e o planejamento da ação, ${ }^{18}$ proporcionando aos trabalhadores a oportunidade de participação. Faz-se essencial identificar as necessidades desse profissional, para saber qual o tema, metodologia e objetivo, entre outros aspectos norteadores da atividade.

No que se refere aos principais temas abordados nas qualificações/ capacitações, os profissionais elencaram as Doenças Crônicas (feridas crônicas, hipertensão, diabetes) e as drogas.

Capacitando sobre drogas, sobre álcool, sobre DST, diabetes $e$ hipertensão. (TE4)

Treinamento pra sala de vacina, a respeito de amamentação, sobre as doenças sexualmente transmissíveis, sobre as pessoas que tem diabetes, os hipertensos, feridas crônicas, queimaduras. (TE1)

Mencionaram ainda, capacitações voltadas aos seguintes temas: amamentação/aleitamento materno; Doenças Sexualmente Transmissíveis (DST); sobre os modelos assistenciais em que atuam (ESF, CAPS); grupos vulneráveis; diagnóstico das populações em situação de vulnerabilidade; sala de vacina; queimaduras; cuidando do cuidador; Programa Nacional de Melhoria do Acesso e da Qualidade da AB; gerenciamento; legislações; gestação de alto risco; violência; dengue; hepatites notificações; entre outras temáticas.

Diante do exposto, os profissionais reconhecem a Educação Continuada e Permanente como importantes estratégias para qualificar a assistência aos grupos populacionais vulneráveis, sugerindo outras estratégias e recursos fundamentais que poderiam ser implantadas nos cenários investigados, como a quantidade suficiente de profissionais, materiais e recursos suficientes para o trabalho e ampliação da equipe, com ações multiprofissionais, além da valorização do profissional, equiparação salarial, permanência/cobertura do profissional na unidade de saúde, resgate de valores, melhorias nas condições de trabalho e na estrutura física dos serviços, implantação de protocolos, melhor relação entre Secretaria de Educação e da Saúde, entre outros. 


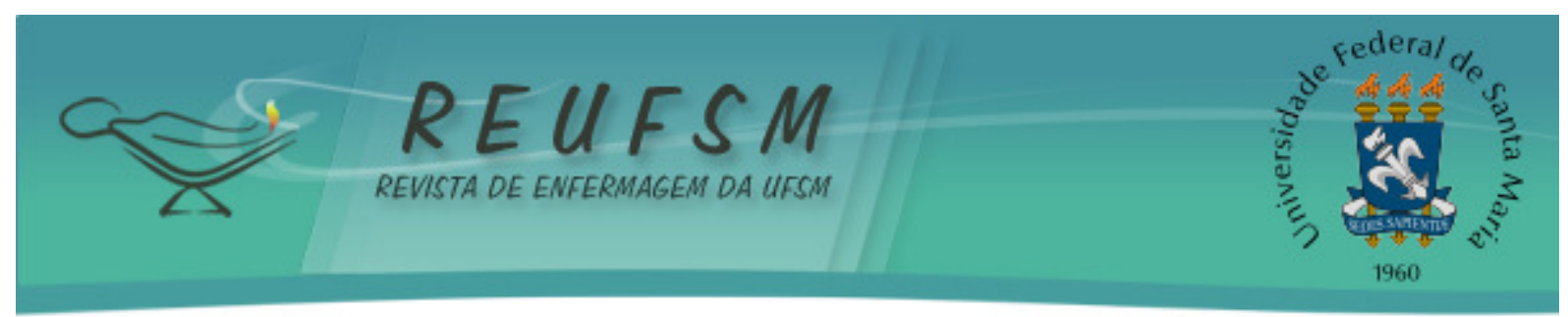

[...] enquanto não for valorizado o profissional como ser humano $e$ ele receber por esse valor, ou por este trabalho prestado [...] a não ser que você se doe bastante, tenha amor a camiseta, que você vai fazer seu trabalho da melhor forma possível. (E1)

\section{A questão multiprofissional seria bem importante para fortalecer o atendimento. (E3)}

Neste contexto, uma pesquisa ${ }^{19}$ sinaliza para a questão dos recursos humanos no processo de mudança da gestão e atenção à saúde. A escassez no quadro de profissionais, precarização das relações de trabalho e falta de compromisso por parte de profissionais da saúde repercutem no processo de consolidação do Sistema ${ }^{19}$ e na assistência despendida aos diferentes grupos.

Da mesma forma, das "novas possibilidades de organização dos processos de trabalho, emergem novas formas de relação entre os trabalhadores, inclusive a necessidade de se trabalhar numa perspectiva interdisciplinar". ${ }^{20: 2146} \mathrm{O}$ trabalho em saúde é, ou deveria ser, um trabalho coletivo, pois acontece mediante a atuação de diversos profissionais da saúde e trabalhadores capacitados, possibilitando por meio da execução de suas atividades a manutenção da estrutura institucional. ${ }^{21}$

Os profissionais ressaltaram que as ações para melhorar o processo de trabalho na $A B$, são viáveis, muitas de baixo custo e poderiam qualificar a assistência aos grupos populacionais vulneráveis, bem como trazer maior satisfação laboral. Apontam ainda, a importância do gestor na viabilização das ações e recursos para qualificação da assistência.

Psicólogo, assistente social, computador e tempo de planejamento não são coisas tão difíceis de organizar e acho que isso aí poderia dar um bom salto de qualidade. (M4)

Depende muito [...] de um olhar da gestão [...] nos três níveis: municipal, estadual e federal. (M1)

Cumpre destacar que são quatro as macro-funções dos gestores em saúde: formulação de políticas e planejamento; financiamento; coordenação, regulação, controle e avaliação das ações, bens, serviços e sistema; e prestação direta de ações. ${ }^{22}$ No entanto, o comprometimento dos trabalhadores da saúde e a participação dos usuários - um dos princípios organizativos do SUS - são importantes e precisam ser analisados e valorizados quando se discute a gestão em saúde.

Portanto, apesar dos avanços na formação na área de saúde, evidenciados neste estudo, há necessidade de maiores subsídios quanto as estratégias de ensino e práticas que visem aprimorar e elevar o grau de conhecimento destes profissionais, qualificando a assistência prestada à população.

\section{CONSIDERAÇÕES FINAIS}

Para compreensão da vulnerabilidade e como atuar qualificadamente frente a esta no contexto da $A B$, é fundamental sua abordagem durante o processo de formação do profissional.

Neste estudo, identificou-se que a discussão sobre promoção da saúde e prevenção de doenças, com foco na saúde da família, foi o meio empregado para abordar a 


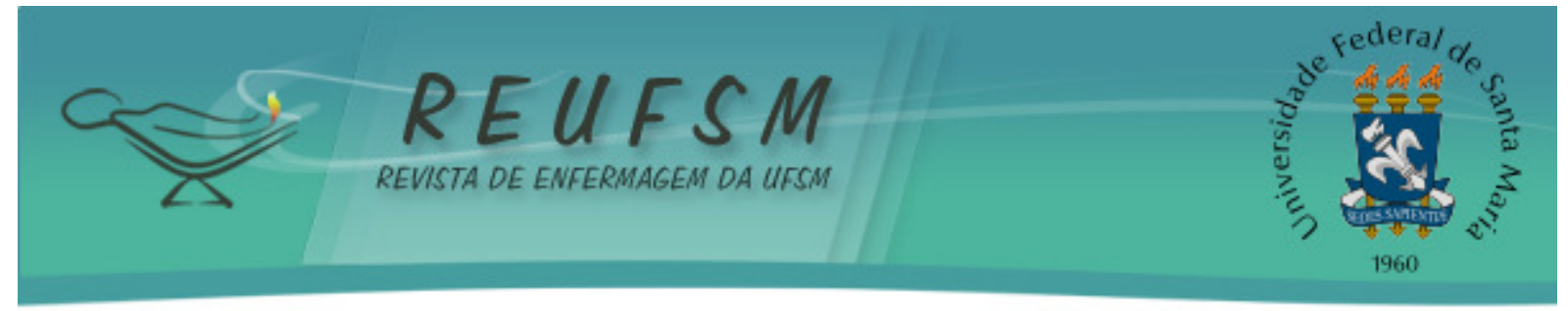

vulnerabilidade na formação dos profissionais. Além do que, a vulnerabilidade é idealizada, sobretudo, como inerente à $A B$.

Os profissionais destacaram a Educação Continuada e Permanente, como alternativas de aprimoramento que poderiam fornecer embasamento científico e troca de saberes, uma vez que a formação profissional mostrou-se frágil no reconhecimento das populações vulneráveis e na proposta de estratégias de atenção.

Apesar da notável progressão na formação acadêmica e técnica, a vulnerabilidade deve ser abordada e discutida no processo de formação dos profissionais, aproximando-os do contexto socioeconômico e cultural dos sujeitos, em busca do cuidado integral e humanizado. Nesse sentido, acredita-se que a integração do meio acadêmico com a comunidade permite a avaliação in loco das necessidades individuais e coletivas, elencando formas de orientação que ampliem a visão futura como profissional coresponsável pela saúde da população de um determinado contexto.

Seguindo essa estratégia, a educação permanente emerge como ferramenta para o aperfeiçoamento do conhecimento dos profissionais de saúde e qualificação da assistência prestada à população, com vistas a evitar ou minimizar a susceptibilidade de indivíduos e grupos à exposição aos agravos/doenças e ao adoecimento. No entanto, cabe salientar a importância da atuação intersetorial quando se busca a redução da vulnerabilidade, tendo em vista que sua gênese conceitual, por si só, já emerge da multifatorialidade.

Considera-se, portanto, que compreender o conceito de vulnerabilidade ajuda também a entender comportamentos assumidos por indivíduos e populações frente aos eventos adversos e às situações mais inesperadas que se apresentam no ciclo da vida, contribuindo para a qualificação do cuidado em saúde.

Constata-se a necessidade de novos estudos com enfoque à temática da vulnerabilidade nas propostas curriculares educacionais das instituições de ensino em saúde, bem como sua metodologia de abordagem, com vistas a identificar e promover estratégias que minimizem ou sanem as possíveis lacunas oriundas do processo formativo.

\section{REFERÊNCIAS}

1. Amaral DP, Oliveira FB. O ProUni e a conclusão do ensino superior: questões introdutórias sobre os egressos do programa na zona oeste do Rio de Janeiro. Ensaio: avaliação e políticas públicas em educação [Internet]. 2011 [acesso em 2014 ago 18];19(70):21-42.

http://www.scielo.br/pdf/ensaio/v19n70/v19n70a03.pdf.

2. Morais Neto OL, Castro AM. Promoção da saúde na atenção básica. Brasília: Ministério da Saúde, Secretaria de Atenção à Saúde, Departamento de Atenção Básica; 2008. (Revista Brasileira Saúde da Família; 17).

3. Ayres JRCM, Calazans GJ, Saletti Filho HC, França Júnior I. Risco, vulnerabilidade e práticas de prevenção e promoção da saúde. In: Campos GWS, Minayo MCS, Akerman M, Drumond Júnior M, Carvalho YM, organizadores. Tratado de saúde coletiva. São Paulo: Hucitec; Rio de Janeiro; Fio Cruz; 2006.

4. Brasil. Ministério da Saúde. Secretaria de Vigilância em Saúde. Secretaria de Atenção à Saúde. Política Nacional de Promoção da Saúde. PNPS: revisão da Portaria MS/GM nº 687, de 30 de março de 2006. $3^{\text {a }}$ ed. Brasília; 2015.

5. Trindade LL, Ferraz L, Zanatta EA, Bordignon M, Mai S, Ferraboli S. Vulnerabilidade na adolescência: a ótica dos enfermeiros da saúde da família. J Nurs Ufpe online [Internet]. 


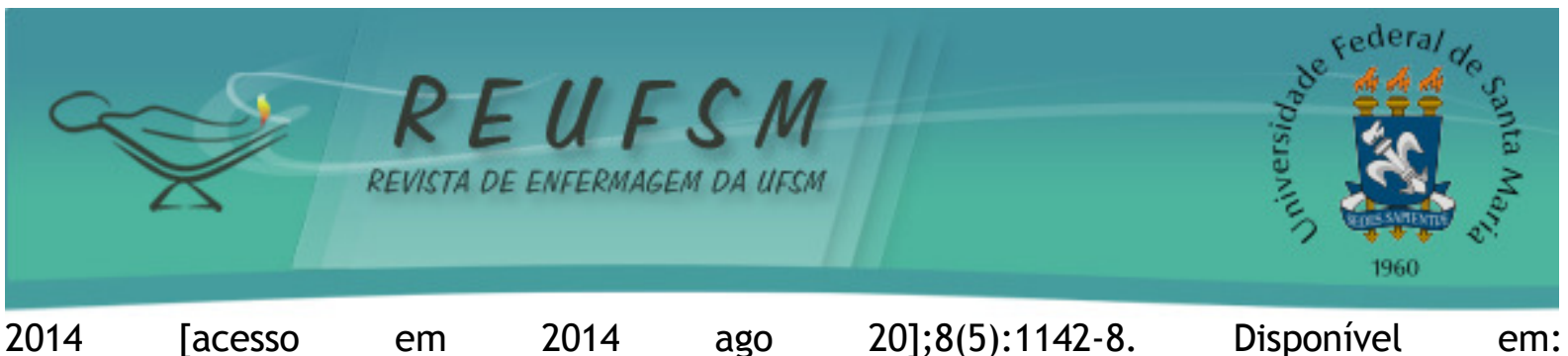
http://www.revista.ufpe.br/revistaenfermagem/index.php/revista/article/view/5919/pdf_ 5012.

6. Batista KBC, Gonçalves OSJ. Formação dos profissionais de saúde para o SUS: significado e cuidado. Saude Soc. 2011;20(4):884-99.

7. Ministério da Saúde. Secretaria de Gestão do Trabalho e da Educação na Saúde. Departamento de Gestão da Educação em Saúde. Política Nacional de Educação Permanente em Saúde. Brasília: Ministério da Saúde; 2009.

8. Silva CT, Terra MG, Roso CC, Souto VT. Educação permanente em saúde: percepção de profissionais de uma residência. Rev Enferm UFSM [Internet]. 2013; [acesso em 2014 mar 23];3(N Esp):627-35. Disponível em: http://cascavel.ufsm.br/revistas/ojs2.2.2/index.php/reufsm/article/view/11067/pdf.

9. Guimarães EM, Martin SH, Rabelo FCP. Educação permanente em saúde: Reflexões e desafios. Cienc Enferm [Internet]. 2010 [acesso em 2014 fev 20]; 16(2):25-33. Disponível em http: / / www.scielo.cl/scielo.php?pid=S0717-95532010000200004\&script=sci_arttext.

10. Bardin L. Análise de conteúdo. Lisboa: Edições 70; 2009.

11. Pinto ICM, Esperidião MA, Silva IV, Soares CM, Santos L, Fagundes TLQ, et al. Trabalho e educação em saúde no Brasil: tendências da produção científica entre 1990-2010. Ciênc Saúde Coletiva [Internet]. 2013 [acesso em 2014 mar 25];18(6): 1525-34. Disponível em: http: / / www.scielo.br/scielo.php?pid=S1413-81232013000600002\&script=sci_arttext.

12. Pagani R, Andrade LOM. Preceptoria de território, novas práticas e saberes na estratégia de educação permanente em saúde da família: o estudo do caso de Sobral, CE. Saúde Soc [Internet]. 2013 [acesso em 2014 mar 22];21(1):94-106. Disponível em: http: / / www.scielo.br/scielo.php?script=sci_arttext\&pid=S0104-12902012000500008.

13. Cardoso IM. "Rodas de educação permanente" na atenção básica de saúde: analisando contribuições. Saúde Soc. 2013;21 Supl 1:18-28.

14. Baraldi S, Díaz MYP, Martins WJ, Júnior DAC. Globalização e seus impactos na vulnerabilidade e flexibilização das relações do trabalho em saúde. Trab Educ Saúde. 2008;6(3):539-48.

15. Souza SNDH, Mello DF, Ayres JRCM. O aleitamento materno na perspectiva da vulnerabilidade programática e do cuidado. Cad Saúde Pública [Internet]. 2013 [acesso em 2014 mar 30];29(6):1186-94. Disponível: http://www.scielo.br/scielo.php?pid=S0102311X2013000600015\&script=sci_arttext.

16. Ferreira MEV, Schimith MD, Cáceres NC. Necessidades de capacitação e aperfeiçoamento dos profissionais de equipes de saúde da família da $4^{a}$ Coordenadoria Regional de Saúde do Estado do Rio Grande do Sul. Cienc Saude Coletiva. 2010;15(5):261120.

17. Murofuse NT, Rizzotto MLF, Muzzolon ABF, Nicola AL. Diagnóstico da situação dos trabalhadores em saúde e o processo de formação no polo regional de educação permanente em saúde. Rev Latinoam Enferm. 2009;17(3):314-20.

18. Ferreira JCOA, Kurcgant P. Directors of nursing point of view of the Professional capacitating program for nurses working in major teaching medical centers. Acta Paul Enferm [Internet]. 2009 [acesso em 2014 mar 3];22(1):31-6. Disponível em: http://www.scielo.br/pdf/ape/v22n1/en_a05v22n1.pdf. 


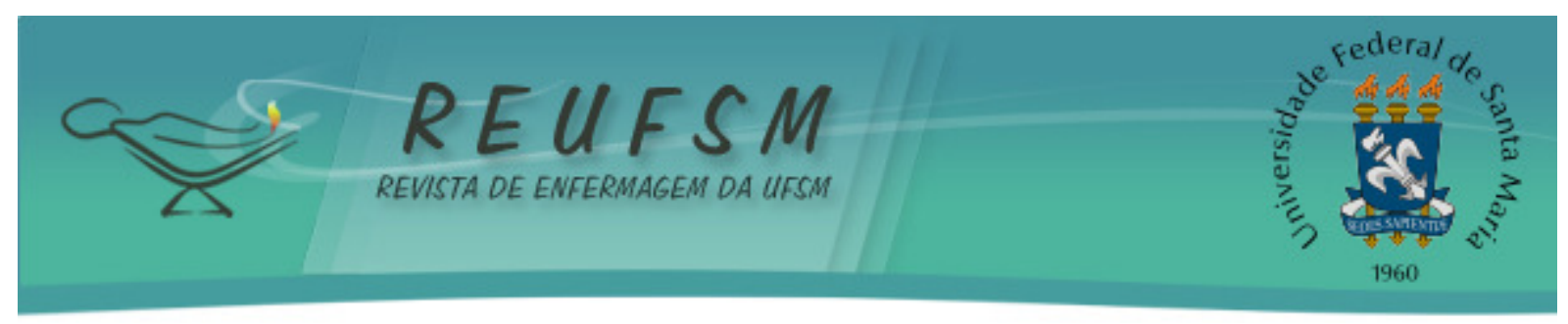

19. Pinto ICM, Teixeira CF. Formulação da política de gestão do trabalho e educação na saúde: o caso da Secretaria Estadual de Saúde da Bahia, Brasil, 2007-2008. Cad Saúde Pública [Internet]. 2011 [acesso em 2014 mar 23];27(9):1777-88. Disponível em: http: / / www.scielo.br/scielo.php?pid=S0102-311X2011000900011\&script=sci_arttext.

20. Guimarães JMX, Jorge MSB, Assis MMA. (In)satisfação com o trabalho em saúde mental: um estudo em Centros de Atenção Psicossocial. Cienc Saude Colet. 2011; 16(4):2145-54.

21. Leopardi MT, Kirchhof AL, Capella BB, Pires DP. O processo de trabalho em saúde: organização e subjetividade. Florianópolis: Papa-Livros; 1999.

22. Noronha JC, Lima, LD, Machado CV. A gestão do Sistema Único de Saúde: características e tendências. In: Anais da $2^{\text {a }}$ Conferência Nacional de Ciência, Tecnologia e Inovação em Saúde; 2004 jul. 25-28; Brasília, DF, Brasil. Brasília (DF): Ministério da Saúde; 2005. p. 45-94.

Data de recebimento: 07/05/2014

Data de aceite: 01/06/2015

Contato com autor responsável: Letícia de Lima Trindade

Endereço postal: Rua Fernando Machado, 685D, ap. 1103, Centro, CEP: 89802-111, Chapecó, SC, Brasil.

E-mail: letrindade@hotmail.com 